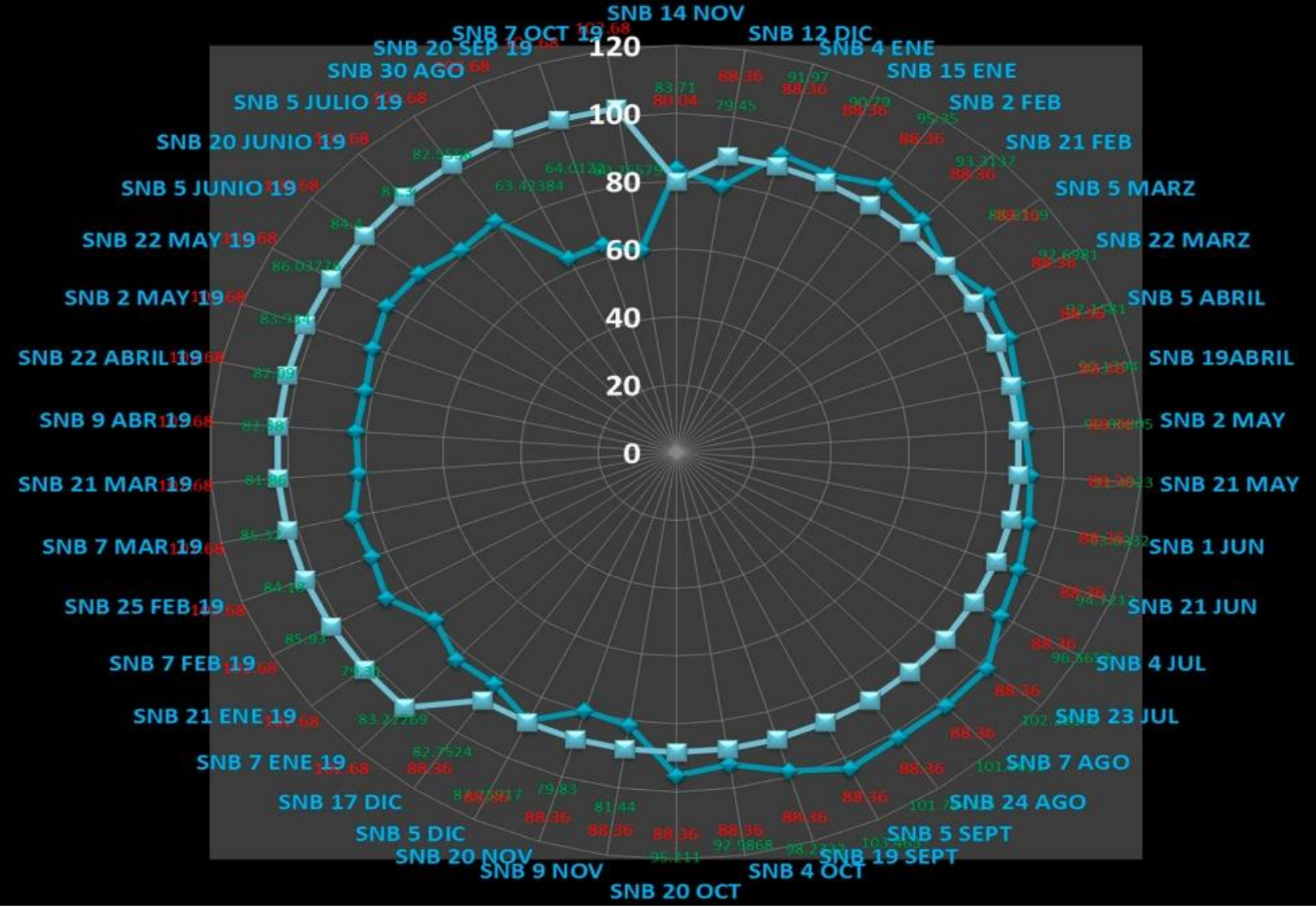

(c) Alberto García Leyva. 2019.

\title{
Un año de la Sustracción Nominal Básica (SNB), imagen del escenario económico-social de Xalapa, Veracruz. Una propuesta práctica del OBSERVES-IIESES-UV
}

Alberto García Leyva ${ }^{1}$

RESUMEN: El observatorio de Economía y Sociedad OBSERVES, surge por la necesidad de generar información novedosa y pertinente, con la misión de acercar información para su uso, debate y análisis al público en general y se encarga de monitorear la evolución de una serie de indicadores económicos-sociales para la ciudad de Xalapa, entre las cuales se encuentran la Sustracción Nominal Básica SNB, indicador capaz de medir los alcances de un salario mínimo con respecto a una alimentación saludable, los resultados se han vigilado por un período mayor a un año, se presentan cambiantes y mayoritariamente adversos, pero en los últimos periodos ha presentado una mejoría, la SNB de Xalapa también refleja el sentir de la sociedad del país.

\footnotetext{
${ }^{1}$ Instituto de Investigaciones y Estudios Superiores Económicos y Sociales (IIESES), Universidad Veracruzana, contacto: albergarcia@uv.mx/albertogarcialeyva12@hotmail.com
}

UVserva No. 8 Octubre 2019 - marzo 2020, ISSN: 2448-7430.

Recepción: 28/agosto/2019

Aceptación: 16/octubre/2019

DOI: $10.25009 /$ uvserva.v\%vi\%i.2632 
Artículo - Observatorio de Economía y Sociedad

Palabras clave: Observatorio, economía, sociedad, Sustracción Nominal Básica, indicadores.

ABSTRACT: The Observatory of Economy and Society OBSERVES, arises from the need to generate novel and relevant information, with the mission of bringing information for use, debate and analysis to the general public and is responsible for monitoring the evolution of a series of economic indicators- Social for the city of Xalapa, among which are the Basic Nominal Subtraction SNB, indicator capable of measuring the reach of a minimum wage with respect to healthy eating, the results have been monitored for a period greater than one year, are presented changing and mostly adverse, but in recent periods has shown an improvement, the SNB of Xalapa also reflects the feeling of society in the country.

Keywords: Observatory, Economy, Society, Basic Nominal Subtraction, Indicators.

\section{El observatorio de Economía y Sociedad de la Universidad Veracruzana, ejes y acciones para entender el entorno socio-económico de Veracruz}

$\square$ 1 observatorio de Economía y Sociedad de la Universidad Veracruzana (OBSERVES) nació por la necesidad de generar información para el público en general pero enfocado al uso académico, sobre todo para los estudiantes y profesores, información también útil para evidenciar la realidad contextual en la que se puede desarrollar la vida de una persona. Es un esfuerzo para poner al alcance de los usuarios información pertinente y actualizada sobre el escenario en un mundo cotidiano, con información realista, palpable y fácilmente comprobable y que además está representada con las experiencias que viven las personas de la ciudad de Xalapa que se pueden capturar en el desarrollo y convivencia diaria entre sus ciudadanos.

El observatorio está compuesto por una serie de ejes temáticos (Gastos en la familia en Xalapa, Temas de coyuntura e Información satélite) enfocados a la generación de información novedosa y enfocada a ser de gran utilidad para diferentes disciplinas, el primero de estos ejes temáticos, corresponde al indicador base del observatorio; la Sustracción Nominal Básica (SNB), así como sus complementos enfocados en el gasto de la familia, a través de unos indicadores noveles que intentan mostrar la realidad económica en la cual se ve envuelta una persona, que percibe un salario mínimo diario y que muestran de manera indirecta los diferentes fenómenos y consecuencias en los que se desarrolla la 


\section{Artículo - Observatorio de Economía y Sociedad}

sociedad mexicana, enfocados a los habitantes de la ciudad de Xalapa en el estado de Veracruz, estos indicadores se mostrarán a detalle más adelante en este documento.

De manera general, los ejes temáticos del observatorio abarcan tres grandes temas; el primero sobre la Sustracción Nominal Básica y complementos, el segundo eje temático relativo a temas de coyuntura, y el tercer sector referente a información satélite. Estos ejes temáticos tienen los objetivos de generar información cuya fuente primaria sea oficial y confiable así como generar temas de interés para la sociedad. La información es publicada en forma de boletines que sean de fácil comprensión y que resuman una gran cantidad de información en atractivas infografías, también tiene el objetivo de poder llegar a ser un repositorio de diversos proyectos que se elaboran en el Instituto de Investigaciones y Estudios Superiores Económicos y Sociales de la Universidad Veracruzana (IIESES), para cumplir con estos objetivos se realizan una serie de acciones que consiste en calcular los indicadores base y generar la información, previo a una depuración de bases de datos y registros (OBSERVES, 2018).

\section{OBSERVES, planteamiento y contexto en el que se desarrolla la SNB}

La tarea primordial de OBSERVES es la de vigilar la evolución de una serie de indicadores a través del tiempo, estos indicadores corresponden a una problemática que se presenta en la sociedad xalapeña y que es representativa del estado presente a nivel nacional: el alcance de un salario mínimo.

El objetivo final que persigue el vigilar la evolución de los indicadores OBSERVES es medir la calidad de vida de las personas, de medir el sendero para desarrollar una vida digna y de cómo este objetivo particular representa líneas de acción a nivel social y comunitario. La calidad de vida corresponde a la incorporación de las diferentes pautas individuales a una representación social, como lo señalan Mercado y Nava, 2013.

Capturar la calidad de vida o el sendero para desarrollar una vida digna no es tarea fácil, ya que esta acción deberá presentar el objetivo de manera multidimensional, involucrando a las diferentes aristas que puede traer una problemática social de tal magnitud. Para poder evaluar la calidad de vida, se debe contemplar el factor multidimensional, que agrega conceptos como; el estado económico, la educación, el empleo, estilo de vida y la vivienda (Avila, Carlos; Velarde, 2002).

UVserva No. 8 Octubre 2019 - marzo 2020, ISSN: 2448-7430.

Recepción: 28/agosto/2019

Aceptación: 16/octubre/2019

DOI: 10.25009/uvserva.v\%vi\%i.2632 
El primero de estos indicadores se denomina Sustracción Nominal Básica (SNB), que intenta medir los alcances de un salario mínimo frente al gasto que tiene una persona para tener una alimentación saludable que le permita tener una vida digna, a través de una canasta de productos que son considerados saludables, se calcula el precio nominal en la actualidad y se compara con el salario mínimo diario para una persona, la SNB está diseñada con un grado de adaptabilidad para cualquier ciudad de la República Mexicana siempre y cuando se tenga con información primaria pertinente, los resultados de este indicador pueden desarrollar una serie de investigaciones que giran en torno a la situación económica de las familias de una ciudad determinada, y contempla niveles tanto de precios como de ingresos que son palpables en la sociedad, además representa una parte de la realidad en la población (García, 2018).

El indicador antes expuesto surge como respuesta a una problemática que se da en la sociedad, enfocada principalmente a situaciones económicas y de salud, sobre todo aquellas referentes la alimentación saludable. Como mencionan en su investigación Mundo-Rosas, Verónica; Shamah-Levy, Teresa; Rivera-Dommarco y Grupo de Seguridad Alimentaria en México, 2013; Casi en un tercio de los hogares en México han reducido la cantidad y calidad de alimentos que consumen o sufrido hambre, más del 40\% de los hogares mexicanos entra en la categoría de inseguridad alimentaria leve (hogares que se preocupan para que los alimentos duren en el mediano y corto plazo), situación ocasionada por la falta de recursos económicos, por lo que ante esta carencia las personas empiezan a consumir alimentos deficientes en micronutrimentos y altos en grasas saturadas y azúcares.

Dicho lo anterior también surgió la necesidad de calcular el alcance del salario mínimo pero con una alimentación que fuera típica de la sociedad xalapeña por lo que desde el observatorio se contempló la creación de un indicador hermano a la SNB capaz de medir este fenómeno económico y social.

Surgen así la Sustracción Nominal Típica SNT, un indicador con la capacidad de expresar la dieta típica de un persona promedio (mercancías de alta demanda en las familias xalapeñas y sus alrededores, según estadísticas), capturando los niveles de precios actuales que se pueden encontrar en los mercados, es decir, el precio nominal de los productos de este tipo de dieta, este indicador tiene una alta representatividad del estado económico y nutricional de la ciudad de Xalapa en la actualidad (Gómez, 2018). 


\section{Artículo - Observatorio de Economía y Sociedad}

Otro de los sectores de suma importancia para tratar de medir la calidad de vida de las personas, es aquel que hace referencia el precio del agua potable que es consumido en las viviendas, el estado en el cual se encuentra el gasto que representa el consumo de un recurso vital, es un escenario que se debe procurar medir y vigilar la evolución que puede llegar a tener. Cómo es típico en este tipo de fenómenos, esta problemática también encuentra su factor multidimensional, entre las que se pueden señalar las condiciones en las que se encuentran un determinado sector de viviendas, sobre todo en las condiciones económicas y cómo éstas afectan la calidad de vida de las personas que habitan estás viviendas. Existe la necesidad de desarrollar unidades de medición que funcionen para visualizar las circunstancias de hábitat que se puedan encontrar, esto toma fuerza a partir de una falta de información sobre la calidad de vida, comportamiento y satisfacción de los usuarios de las viviendas (Hernández, Gustavo; Velásquez, 2014).

La falta de información pertinente necesaria para utilizarse en diversas investigaciones, impulsó a la formulación del indicador denominado Proporción Salarial Hídrica Doméstica (PSHD) que tiene el objetivo de medir el valor económico del consumo de agua que se tiene en un hogar en la ciudad de Xalapa, también tiene el objetivo de aportar nuevos conceptos y metodologías en torno al uso de este importante bien, tomando en consideración el precio de la tarifa del consumo de este recurso de manera periódica (García Leyva, 2018).

Estos tres indicadores de OBSERVES se monitorean cada determinado tiempo de manera mensual o quincenal dependiendo el indicador, todos en su conjunto muestran la realidad social y económica de la ciudad de Xalapa y que puede ser representativo de la situación a nivel nacional, para mostrar el escenario actual presentado en el último año 2018, en este artículo de investigación se mostrará la evolución de la SNB misma que se detalla a continuación.

\section{La SNB, desarrollo y evolución para el año 2018}

La Sustracción Nominal Básica, como se mencionó anteriormente, estima la evolución de precios de una canasta considerada saludable en comparación con el salario mínimo, la suma de los precios nominales actuales de una serie de productos idóneos listados por la Secretaría de Salud (con productos como zanahoria, plátano, jitomate, cebolla, chile, nopal, sandía, limón, naranja, tortilla, arroz, papa, huevo, sardina, frijoles, leche, entre otros) en una de sus publicaciones, son sumados y multiplicados por la porción que normalmente

UVserva No. 8 Octubre 2019 - marzo 2020, ISSN: 2448-7430.

Recepción: 28/agosto/2019

Aceptación: 16/octubre/2019

DOI: $10.25009 /$ uvserva.v\%vi\%i.2632 
consume una persona promedio, se calcula el precio de la actualidad con base a la publicación de la Procuraduría Federal del Consumidor (PROFECO) y se determina el Gasto Diario Nutrimental, posteriormente al salario mínimo se le resta el valor del Gasto Diario Nutrimental (García, 2018). Un resultado negativo en la Sustracción Nominal Básica significa que el Gasto Diario Nutrimental es mayor que el salario mínimo, en otros términos, si una persona desea tener una alimentación sana durante un día, está tendría que endeudarse con tal de cumplir tal requerimiento, de lo contrario al existir una SNB positiva se tendría el escenario de que sobra un monto del salario mínimo diario para el resto de los gastos personales.

En la Gráfica número 1 se puede observar la comparación entre el Gasto Diario Nutrimental y el salario mínimo a través de una gráfica de red. Cómo se puede observar el área del círculo de esta gráfica, es mayor en la que corresponde a la del Gasto Diario Nutrimental, mientras que para la del salario mínimo por algunos sectores es más contraída, lo cual significa que las personas tendrían que endeudarse en ese lapso de tiempo, para cumplir con los requerimientos de tener una dieta saludable, la gráfica muestra la evolución desde el 14 de noviembre del año 2017 hasta el último período de análisis en diciembre del 2018, la sucesión nominal básica se calcula dos veces por mes y su publicación en la página web de OBSERVES es determinada por la información primaria sobre todo de la PROFECO, el salario mínimo es el publicado por la Secretaría de hacienda y crédito Público (SHCP).

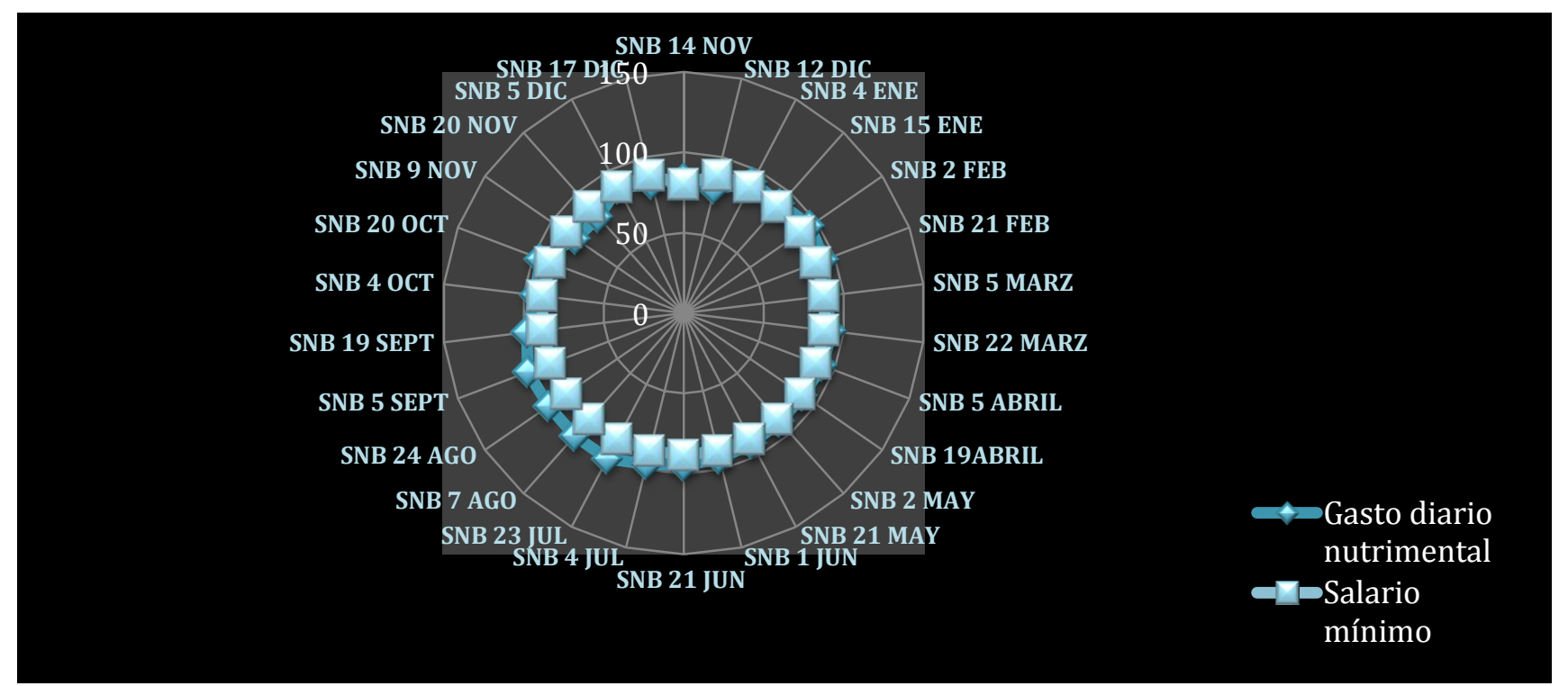

Figura 1. Gasto Diario Nutrimental y salario mínimo en Xalapa (pesos mexicanos). Noviembre 2017-Diciembre 2018. Fuente: Observatorio de Economía y Sociedad del IIESES-UV (OBSERVES- IIESES-UV), disponible en la página web; https://www.uv.mx/observes/general/sustraccion-nominal-basica-snb/. 


\section{Artículo - Observatorio de Economía y Sociedad}

Como se observa en la figura 2 número dos que se muestra a continuación, la evolución de la Sustracción Nominal Básica en Xalapa ha sido en su mayoría negativa, de todos los resultados disponibles solamente existen cinco periodos de análisis positivos, los periodos de análisis en donde la SNB resultó positiva son para el período de diciembre del 2017 y los últimos dos meses del año 2018, la Sustracción Nominal Básica encuentra su resultado más adverso en los meses de agosto, septiembre y octubre, alcanzando el mínimo resultado de \$15.10 de endeudamiento, para mediados de septiembre del 2018 el mejor resultado fue para el mes de diciembre del 2017 con $\$ 8.90$ debido a un aumento en el salario mínimo.

\section{SNB}

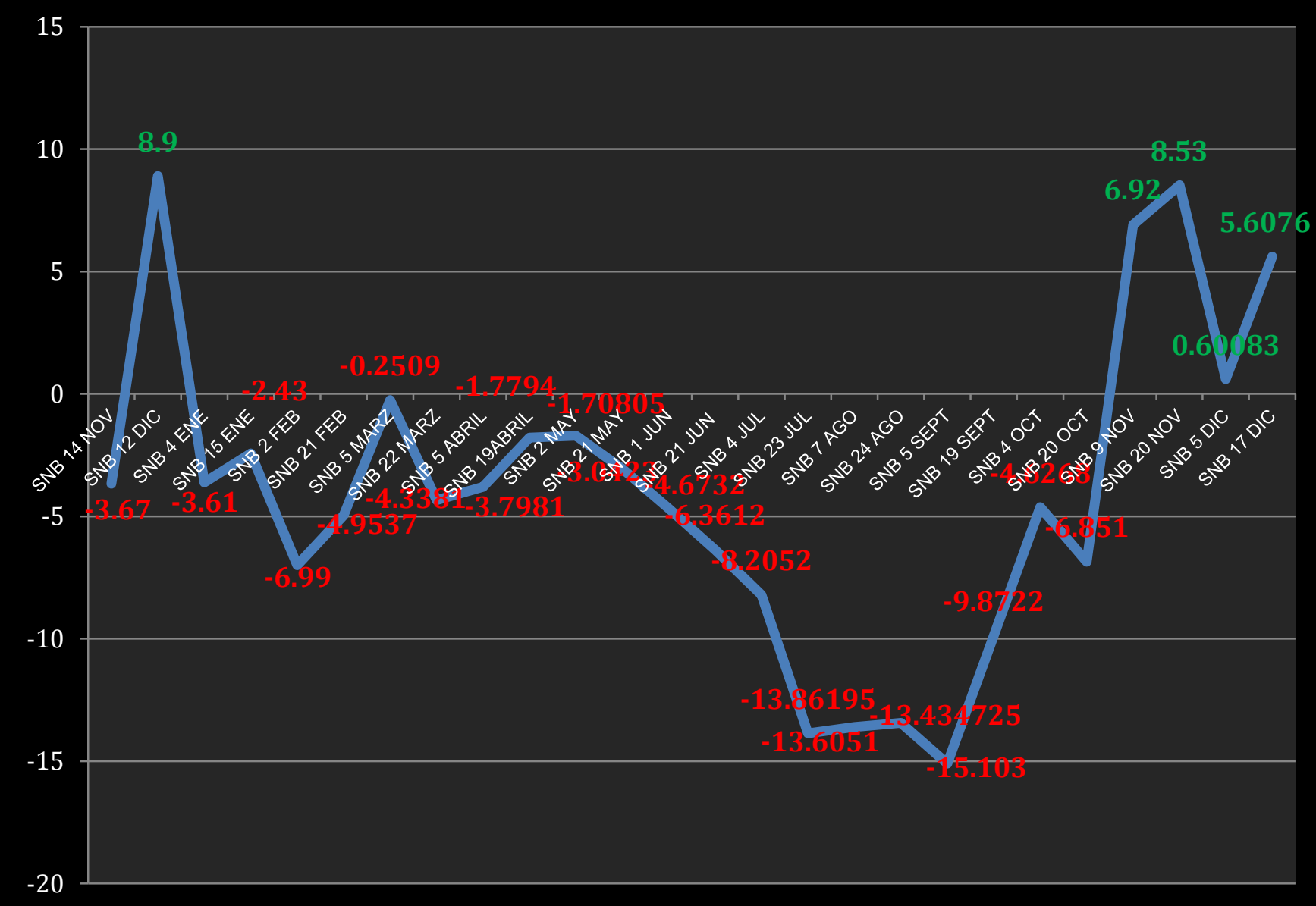

Figura 2. Sustracción Nominal Básica de Xalapa (pesos mexicanos). Noviembre 2017-Diciembre 2018. Fuente: Observatorio de Economía y Sociedad del IIESES-UV (OBSERVES- IIESES-UV), disponible en la página web; https://www.uv.mx/observes/general/sustraccion-nominal-basica-snb/. 


\section{Artículo - Observatorio de Economía y Sociedad}

Si bien la Sustracción Nominal Básica tiene un comportamiento negativo y brusco, se puede llegar a la conclusión que en los últimos dos meses ha tenido una evolución positiva, se ha llegado a recuperar (debido a una reducción en el Gasto Diario Nutrimental, el precio de algunos productos se reduce), en un lapso de tiempo de dos meses se puede encontrar dos de los resultados más extremos de la SNB, el mes de septiembre el resultado más bajo y para el mes de noviembre el segundo resultado más alto del periodo de análisis.

\section{El contexto nacional a través de los números}

La Sustracción Nominal Básica así como los indicadores que vigila el Observatorio de Economía y Sociedad, están desarrolladas en un contexto nacional complicado económicamente, los indicadores de OBSERVES se encuentran principalmente en estados negativos, cómo se vio en el caso de la Sustracción Nominal Básica, la mayoría del tiempo de análisis es negativa, aunque en los últimos meses ha tenido una evolución positiva, para el caso de los otros dos indicadores, encontramos resultados diversos, para la Proporción Salarial Hidrica Doméstica (PSHD) existen resultados adversos que significan un escenario complicado para las personas y cómo se explicó con anterioridad, la problemática individual de las personas se ve reflejada en las problemáticas sociales agregadas, bajo este primicia se muestra en esta sección el contexto nacional a través de información del INEGI, qué hace referencia a la situación de los últimos años en México. En materia de población, México cuenta con un total de 119, 938, 473 personas para el año 2015, el grado promedio de escolaridad de la población de 15 años y más para el año 2015 era de 9.2 y existen un total viviendas particulares habitadas para el año 2015 de 31,949,709 (INEGI, 2018).

Para el caso de la actividad industrial en México, se puede encontrar que la electricidad, gas y agua, en cifras desestacionalizadas mensuales para octubre de 2018, tiene una variación porcentual del 5\% anual lo que coloca a este sector como el aquel que tiene mayor variación porcentual positiva, en comparación con los demás sectores, por otro lado la minería representó una variación anual de $-5.5 \%$ y es el que se encuentra con el peor resultado. El indicador global de la actividad económica tiene una variación porcentual anual del 2.1, el consumo privado en el mercado interior tiene $2.8 \%$ de variación Anual mientras que la inversión fija bruta es de apenas 0.2 \% anual, el índice global de productividad laboral de la economía tiene un -0.8 \% de variación anual (INEGI, 2018).

Mencionado lo anterior se puede encontrar una relación no significativa entre la información expuesta y el comportamiento de la SNB, ya que por un lado se observa una relativa mejoría en indicadores de actividad económica, pero una contracción en materia de productividad laboral, en discordancia con el comportamiento raudo y mayoritariamente 


\section{Artículo - Observatorio de Economía y Sociedad}

negativo de la SNB. Por el momento, no existe evidencia de variables que puedan tener un efecto de causalidad con la SNB.

Uno de los indicadores contextuales más relevantes para el OBSERVES es el Índice Nacional de Precios al Consumidor (INPC) ya que este índice es el encargado oficial de registrar los niveles de precios de los productos de la canasta básica en México, para el caso de OBSERVES la SNB y SNT surgen como respuesta al tratar de medir los niveles de precios para una canasta de productos saludables y de productos típicos consumidos en la ciudad de Xalapa, y visualizar su comportamiento a través del tiempo, indicadores donde los niveles de precios son verídicos y reales que se ven reflejados en los resultados y en el sentir de la población.

El Índice Nacional de Precios al Consumidor (INPC) para el mes de julio del 2018 tenía una variación porcentual del $4.8 \%$ y una acumulada del 1.6\% según datos del INEGI, con estos resultados se puede encontrar ciertas coincidencias con la SNB, sobre todo en el sentido de que existe un aumento sostenido y generalizado de los precios de ciertos bienes, aunque se debe recalcar que para el caso de los indicadores de OBSERVES éstos son calculados y monitoreados de manera constante y frecuente, dos veces por mes, y se puede visualizar una mayor variación en los resultados entre sí, el INPC que se tomó en esta ocasión es la variación porcentual anual, mientras que para el caso de la SNB que se muestra en las gráficas anteriores, corresponden a todos los periodos de análisis que se han realizado en el observatorio. El comportamiento del INPC, en los meses en los que la SNB presentó una mejoría, fue ascendente según datos del INEGI.

\section{Conclusiones}

El Observatorio de Economía y Sociedad OBSERVES, cumple una función fundamental dentro de una institución Universitaria, la de proveer información para el público en general, esta averiguación es novedosa, cumple con los requerimientos científicos y es publicada de manera que pueda ser de fácil comprensión y por ende de fácil aplicación a diversos proyectos de investigación, en apenas algunos meses de monitoreo de un conjunto de indicadores sociales y económicos se ha podido evidenciar una serie de fenómenos que giran en torno a la gastos de las familias en Xalapa y a los alcances del salario mínimo, es aquí donde surgen una serie de directrices y lineamientos en los cuales se pueden aplicar diferentes métodos de entendimiento provenientes de diferentes disciplinas.

UVserva No. 8 Octubre 2019 - marzo 2020, ISSN: 2448-7430.

Recepción: 28/agosto/2019

Aceptación: 16/octubre/2019

DOI: 10.25009/uvserva.v\%vi\%i.2632 


\section{Artículo - Observatorio de Economía y Sociedad}

Para el año 2019 se espera que en los primeros periodos de análisis se tenga una SNB favorecedora, esto por el aumento al salario mínimo que entrará en vigor a partir de enero del año ya mencionado, ante esta circunstancia se tiene que contemplar la evolución de este indicador a través del tiempo y del transcurso del año, ya que como es común, se espera un aumento generalizado en los productos que son monitoreados, por lo que puede ocasionar un aumento recíproco del Gasto Diario Nutrimental, lo que ocasionaría una SNB proporcional y/o equivalente a la experimentada durante el año 2018.

La situación social y económica de la ciudad de Xalapa a través de los resultados de la SNB se puede visualizar en un contexto sumamente cambiante en su mayoría adverso, pero con resultados positivos en los últimos periodos de análisis, bajo este aspecto se puede concebir un cierre de año favorecedor para las circunstancias en las que se desarrolla la sociedad, a juzgar por la evolución de los periodos de análisis, sin embargo no es suficiente para otorgarle a una persona y a una familia una vida digna, para que exista una mejoría en los resultados del indicador, es necesario un aumento al salario mínimo y que los precios de los productos básicos para una alimentación saludable se mantengan.

Se puede abonar mencionando la importancia de consumir los productos producidos de manera local, que en ocasiones suelen ser más baratos, ya que en los precios no están integrados factores tales como el transporte, precio de la gasolina que influye directamente en los precios de los bienes, aranceles, si el producto viene de otro país, e intermediarios.

Se concluye que para la situación de Xalapa y sus alrededores, la ventaja geográfica en la posibilidad de adquirir productos a precios accesibles, esto ya que la ciudad capital del estado de Veracruz, se encuentra localizada entre el puerto de Veracruz, uno de los más importantes del país, y a una distancia relativamente cerca de importantes ciudades medulares tales como la Ciudad de México y Puebla, y por ende de los grandes centros de abastecimiento y distribución nacional de alimentos, presionando así a una SNB con resultados ventajosos.

A partir de esta observación geográfica local, surge una recomendación para los consumidores específicos, que versa sobre adquirir los productos saludables en centros mayoristas y centrales de abastos de la ciudad de Xalapa, donde los precios son más bajos y la calidad del producto puede ser mejor. 
Es importante aplicar el estudio de la SNB para otras ciudades del estado de Veracruz, para visualizar cual es el comportamiento del indicador en diferentes contextos pero dentro de la región, ya que el estado Veracruzano tienen una geografía amplia que puede reflejar diferentes situaciones, importante también es medir la SNB para ciudades del resto de la República Mexicana y su comportamiento atreves del tiempo, ya que el indicador fue diseñado para su adaptación para cualquier ciudad del mundo y para fines comparativos.

La SNB también cuenta con elementos aplicables y adaptables para medir las diferentes circunstancias estipuladas en los Objetivos del Desarrollo Sostenible, y sirve como mecanismo evaluador para las políticas públicas del desarrollo sustentable, función que también es propia de un observatorio universitario. 
Artículo - Observatorio de Economía y Sociedad

\section{Referencias}

Avila, Carlos; Velarde, E. (2002). Evaluación de la calidad de vida. Salud Pública de México, 44(4), 349-361. Retrieved from http://www.insp.mx/salud/index.html

García, Alberto. (2018). Indicador de precios, gastos y poder adquisitivo nominal entorno a un consumo alimenticio saludable. Una propuesta desde el Observatorio de Economía y Sociedad de la Universidad Veracruzana. UVServa. Revista electrónica de la Coordinación Universitaria de Observatorios, (5), 85-90.

García Leyva (2018). Proporción Salarial Hídrica Doméstica, propuesta de indicador entorno al gasto básico personal. Interconectando Saberes, 3(5), 75-85. Disponible en internet http://is.uv.mx/index.php/IS/article/view/2549/4466

Gómez, K.M. (2018). Costos monetarios de la alimentación actual del mexicano promedio, el caso de Xalapa, Veracruz. Construcción de la Sustracción nominal Típica. UVServa. Revista electrónica de la Coordinación Universitaria de Observatorios, (5), 91-101.

Hernández, Gustavo; Velásquez, S. (2014). Vivienda y calidad de vida. Medición del hábitat social en el México occidental. Revista Bitácora Urbano Territorial, 24(1), 1-36. Retrieved from https://wWW.redalyc.org/articulo.oa?id=74830875016

INEGI. (Instituto Nacional de Estadística y Geografía). Índice Nacional de Precios al Consumidor (INPC). Base 2a Quincena Julio 2018. Recuperado el 18 de Septiembre del 2019 de la página web;

https://www.inegi.org.mx/programas/inpc/2018/default.html\#Datos_abiertos

INEGI. (Instituto Nacional de Estadística y Geografía). México en cifras. Estados Unidos Mexicanos. Recuperado el 27 de diciembre del 2018 de la página web; https://www.inegi.org.mx/app/areasgeograficas/?ag=00

Mercado-Salgado, Patricia; Nava-Rogel, R. M. (2013). Calidad de vida y expectativas de migración en jóvenes de zonas rurales del Estado de México. Población Y Salud En Mesoamérica, 10(2), 1-19. Retrieved from

https://www.redalyc.org/articulo.oa?id=44625652004

Mundo-Rosas, Verónica; Shamah-Levy, Teresa; Rivera-Dommarco, J. A; Grupo de Seguridad Alimentaria en México. (2013). Epidemiología de la inseguridad alimentaria en México. Salud Pública de México, 55(2), S206-S213. Retrieved from

https://www.redalyc.org/articulo.oa?id=10628331018

Observatorio de Economía y Sociedad del IIESES-UV (OBSERVES- IIESES-UV), disponible en la página web; https://www.uv.mx/observes/general/sustraccion-nominal-basica-snb/y boletín descargado el 18 de diciembre del 2018 de la página web; 
https://www.uv.mx/observes/files/2018/12/SNB-2018-24.pdf, Autor del boletín; Alberto Garcia Leyva.

OBSERVES, 2018. Observatorio de Economía y Sociedad del IIESES-UV. Ejes estratégicos. Ejes temáticos. Consultado el 25 de Diciembre del 2018 de la página web; https://www.uv.mx/observes/presentacion/ejes-estrategicos/ 